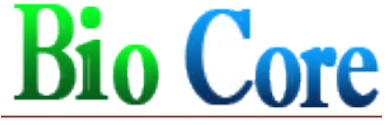

Exploring Scientific Community

\section{International Journal of Nutritional Science}

\section{and Food Technology}

\title{
Herbs and Spices for Diabetes
}

Dragan Jovanov*

Mit University - Skopje, Macedonia, Environmental Resources and Food Security Management

Corresponding Author: Dragan Jovanov, Mit University - Skopje, Environmental Resources and Food Security Management,Tel: +38970867356, Macedonia. Email: daci.j@hotmail.com

Citation: Dragan Jovanov (2017), Herbs and Spices for Diabetes. Int J Nutr Sci \& Food Tech. 3:1, 15-18.

DOI: $10.25141 / 2471-7371-2017-1.0026$

Copyright: (C2017 Dragan Jovanov. This is an open-access article distributed under the terms of the Creative Commons Attribution License, which permits unrestricted use, distribution, and reproduction in any medium, provided the original author and source are credited

Received February 21, 2017; Accepted March 1, 2017; Published March 17, 2017

Diabetes mellitus (DM), commonly referred to as diabetes, refers to a group of metabolic diseases that affect how your body uses blood sugar (glucose).

According to International Diabetes Federation statistics from 2014, an estimated 387 million people worldwide have diabetes. About 90 percent of them have Type 2 diabetes.

There are three main types of diabetes: Type 1, Type 2 and gestational diabetes.

- In Type 1 diabetes, the pancreas fails to produce enough insulin. The exact cause is unknown, however risk increases with certain factors like family history, genetics, exposure to certain viruses like the Epstein-Barr virus, and vitamin D deficiency.

- Type 2 diabetes begins when cells fail to respond to insulin properly. Factors that put you at a higher risk include obesity, excess abdominal fat, inactive lifestyle, family history, age, high blood pressure, high cholesterol, high triglyceride levels, and a history of polycystic ovaries.

- Gestational diabetes affects women during pregnancy.

Some of the symptoms of high blood sugar include frequent urination, increased thirst or hunger, unexplained weight loss, fatigue, slow healing process and frequent infections. If left untreated, it can cause many complications.

Maintaining normal blood sugar levels is necessary for your overall health. You can do it with a healthy diet, regular exercise, not using tobacco, maintaining a healthy body weight and taking the prescribed medications. You can also try some herbs and spices to ease symptoms and control your blood sugar.

Here are the top 10 herbs and spices for diabetes.

\section{Cinnamon}

Cinnamon contains bioactive components that can help lower blood sugar levels.

International Journal of Nutritional Science and Food Technology An Open Access Journal
A 2013 study published in the Annals of Family Medicine and Diabetes Care shows that cinnamon is beneficial for people with Type 2 diabetes.

Based on this study, it may have a beneficial effect on fasting plasma glucose, LDL cholesterol, HDL cholesterol and triglyceride levels in patients with Type 2 diabetes.

- You can take 1 to 2 grams of cinnamon daily in the form of tea. You can also sprinkle it on your oatmeal, smoothies or use it in cooking and baking.

- Another option is to take cinnamon supplements. However, always consult a doctor for the proper dosage suitable for your condition.

Note: If you take a blood-thinning medication, avoid taking cinnamon.

\section{Fenugreek}

Fenugreek is an herb that diabetic people should include in their diets. Due to its hypoglycemic activity, it can improve glucose tolerance and lower blood sugar levels. In addition, its fiber content slows down the absorption of carbohydrates and sugars.

In a 2009 study published in the International Journal for Vitamin and Nutrition Research, researchers concluded there is a positive effect of fenugreek seeds on blood glucose and lipid profiles in Type 2 diabetic patients.

- Soak 1 to 2 tablespoons of fenugreek seeds in water overnight. The next morning, drink the water and eat the seeds on an empty stomach. Follow this remedy daily.

- You can also eat baked goods made with fenugreek flour.

- Another option is to take a supplement after consulting a doctor.

3. Ginger

Anti-diabetic, hypolipidemic and anti-oxidative properties in 
ginger are beneficial in controlling blood sugar levels. Ginger also can improve insulin sensitivity, reduce oxidation and improve cholesterol levels. It even helps with weight loss.

A 2014 study published in the Complementary Therapies in Medicine journal found that daily consumption of three 1-gram capsules of ginger powder for 8 weeks proved useful for patients with Type 2 diabetes.

- Drink 2 to 3 cups of ginger tea daily.

- Also, include fresh or dry ginger in your cooking.

- Another option is to take ginger supplements after consulting your doctor.

\section{Turmeric}

Turmeric is a good spice to control high blood sugar. It has antiinflammatory, antioxidant, anti-atherosclerotic, heart-protecting and weight-reducing actions that help diabetic people manage blood sugar levels and reduce complications from the disease.

A 2013 study published in the Evidence-Based Complementary and Alternative Medicine and Phytomedicine journal highlighted the important role of curcumin, a key component in turmeric, in the prevention and treatment of diabetes and associated disorders.

A 2014 study published in the Journal of Diabetes \& Metabolic Disorders clearly indicates the beneficial role of turmeric in controlling kidney-related complications caused by diabetes.

- Include raw or powdered turmeric in your cooking.

- Take a supplement, but only after consulting a doctor.

\section{Garlic}

Garlic also helps maintain healthy blood sugar levels in diabetic people, thanks to its anti-diabetic and hypolipidemic properties. It contains sulfur compounds that protect against oxidative damage and even high cholesterol.

According to a 2011 study published in the Pakistan Journal of Pharmaceutical Sciences, garlic supplementation with standard anti-diabetic agent provides better blood sugar control in Type 2 diabetics.

A 2014 study published in the Journal of Diabetes \& Metabolic Disorders suggests that garlic can help treat diabetes and related cardiovascular diseases.

- Eat 2 or 3 raw garlic cloves daily on an empty stomach.

- Also, include garlic in your cooking or take a supplement after consulting a doctor.

\section{Curry Leaves}

Aromatic curry leaves may also be useful in managing high blood sugar. Several minerals in this herb help maintain normal glucose level in the blood. In addition, it influences carbohydrate metabolism. It also reduces the risk of oxidative stress.

A 2013 study published in the International Journal of Food and
Nutritional Sciences highlights the hypoglycemic properties of curry leaf powder in controlling the fasting and postprandial blood glucose levels among diabetics.

A 2007 study published in the Journal of Ethnopharmacology indicates that curry leaf extract can help bring down the severity of diabetes.

- Chew a few tender curry leaves daily in the morning on an empty stomach.

- You can also add curry leaf powder to your soups or salads.

\section{Fenugreek}

Fenugreek seeds (trigonella foenum graecum) are high in soluble fibre, which help lower blood sugar by slowing down digestion and absorption of carbohydrates. Several clinical trials showed that fenugreek seeds can improve most metabolic symptoms associated with both type 1 and type 2 diabetes in humans by lowering blood glucose levels and improving glucose tolerance. It contains trigonelline, which is known to reduce blood sugar level. Take the seeds after soaking them in water overnight or powdered form with buttermilk.

\section{Bitter Melon (Karela)}

The fruit contains at least three active substances with antidiabetic properties, including charantin, vicine and an insulinlike compound known as polypeptide-p. Either these substances work individually or together to help reduce blood sugar levels. Bitter melon also contains a lectin that reduces blood glucose concentrations by acting on peripheral tissues and suppressing appetite - similar to the effects of insulin in the brain. This lectin is thought to be a major factor behind the hypoglycemic effect that develops after eating bitter melon. Karela is rich in vitamins a, b1, b2, c, iron and its regular use prevents complication associated with diabetics such as eye complications, neuritis and defective metabolism of carbohydrates.

\section{Jambu fruit (jamun)}

Its seed contains jamboline, which controls the excessive conversion of starch to sugar. Seed powder can be used with water or buttermilk. It reduces the quantity of sugar in urine and allays the unquenchable thirst.

\section{Bael leaves}

They are scientifically proved to be anti-diabetic. Drink fresh juice of leaves with pinch of pepper.

\section{Aloe Vera}

A well known liver tonic, its regular use tones up the hepatic-biliary system and regulates sugar \& fat. Preliminary research suggests that intake of aloe vera juice can help improve blood glucose levels and may therefore be useful in treating people with diabetes. Aloe also have following positive effects due to the presence of compounds such as lectins, mannans and anthraquinones. Decreased blood lipids (fats) in patients that have abnormally high levels of these molecules in their blood. Decreased swelling 
and faster healing of wound injuries: Leg wounds and ulcers are common complications of diabetes, and they typically take longer time to heal than in healthy non-diabetic individuals.

\section{Cabbage}

It is as effective as insulin.

\section{Curry Patta}

Eating 10 fresh fully-grown curry leaves every morning for 3 months is said to prevent diabetes.

\section{Isabgole}

It helps in controlling blood sugar in diabetics by inhibiting the excessive absorption of sugars from the intestines.

\section{Beans}

Beans are high in carbohydrate and fiber and stimulates the production of insulin. It should be eaten liberally to keep diabetes under control.

\section{Cucumber and Radish leaves}

These low a carbohydrate vegetables are beneficial in the prevention and control of diabetes.

\section{Onion}

It has active hypoglycemic substances, $\mathrm{r}$ allyl propyl disulphide $\&$ allicin.

\section{The 15 Best Superfoods for Diabetics}

Include these nutrition superstars in your diabetes diet to lower blood sugar, burn fat, reduce inflammation, and gain more health benefits.

\section{Dark chocolate}

Chocolate is rich in flavonoids, and research shows that these nutrients reduce insulin resistance, improve insulin sensitivity, drop insulin levels and fasting blood glucose, and blunt cravings. But not all chocolate is created equal. In a 2008 study from the University of Copenhagen, people who ate dark chocolate reported that they felt less like eating sweet, salty, or fatty foods compared to volunteers given milk chocolate, with its lower levels of beneficial flavonoids (and, often, more sugar and fat, too). Dark chocolate also cut the amount of pizza that volunteers consumed later in the same day, by 15 percent. The flavonoids in chocolate have also been shown to lower stroke risk, calm blood pressure, and reduce your risk for a heart attack by 2 percent over five years.

\section{Broccoli}

Broccoli is an anti-diabetes superhero. As with other cruciferous veggies, like kale and cauliflower, it contains a compound called sulforaphane, which triggers several anti-inflammatory processes that improve blood sugar control and protect blood vessels from the cardiovascular damage that's often a consequence of diabetes. (Heart disease is the leading cause of death for people with diabetes, so this protection could be a lifesaver.) Sulforaphane also helps flip on the body's natural detox mechanisms, coaxing enzymes to turn dangerous cancer-causing chemicals into more innocent forms that the body can easily release.

\section{Blueberries}

Blueberries really stand out: They contain both insoluble fiber (which "flushes" fat out of your system) and soluble fiber (which slows down the emptying of your stomach, and improves blood sugar control). In a study by the USDA, people who consumed $21 / 2$ cups of wild blueberry juice per day for 12 weeks lowered their blood glucose levels, lifted depression, and improved their memories. Researchers credit these results to anthocyanins in the berries, a natural chemical that shrinks fat cells and also stimulates the release of adiponectin, a hormone that regulates blood glucose levels, among other things. Increasing adiponectin levels can help keep blood sugar low and increase our sensitivity to insulin.

\section{Steel-cut oats}

You may not think of oatmeal as a superfood, but it can help reduce the risk of developing type 2 diabetes. Oatmeal contains high amounts of magnesium, which helps the body use glucose and secrete insulin properly. An eight-year trial showed a 19 percent decrease in type 2 diabetes' risk in women with a magnesiumrich diet, and a 31 percent decreased risk in women who regularly ate whole grains. Steel-cut oats are just as easy to cook as quickcooking oatmeal, but when grains are left whole they are filled with the fiber, nutrients, and bound antioxidants that challenge digestion in a good way, allowing blood sugar to remain more stable.

\section{Fish}

Fish is a slimming star: rich in protein, it will help to keep you satisfied; but also, fish contains a special type of fat that helps cool inflammation. Thousands of studies show that people with the highest blood levels of omega-3 fatty acids have less body-wide inflammation, the very inflammation that leads to and worsens diabetes and weight problems. A fish-rich diet can also reduce your risk of developing health problems, especially stroke, as a result of your diabetes. People who ate baked, broiled, or steamed fish reduced their odds for a stroke by 3 percent, as reported in a 2010 Emory University study. (However, fried fish - such as fastfood fish sandwiches, fish sticks, and fried seafood of any typeincreased risk.)

\section{Olive oil}

Following a Mediterranean-style diet rich in olive oil helps reduce the risk of type 2 diabetes by as much as 50 percent compared to a diet low in fat, according to a recent Spanish study. Independently, researchers at Technical University of Munich (TUM) and the University of Vienna found that olive oil improved satiety the most when compared to lard, butter, and rapeseed (canola) oil. In addition to being a standout source of health-promoting monounsaturated fats, olive oil is also rich in antioxidant nutrients that protect cells from damage, and prevents the development of heart disease. 


\section{Psyllium husk}

This fiber supplement, long used for constipation relief, is proven to help people with diabetes control blood sugar better. A 2010 review from the University of California, San Diego, published in the Annals of Pharmacotherapy, confirms this benefit. People who took psyllium before a meal saw their post-meal blood sugar levels rise 2 percent less than those who didn't use the supplement. One caution: The researchers recommend waiting at least four hours after taking psyllium before taking medications, because psyllium can decrease their absorption.

\section{Cannellini beans}

Packed with protein and cholesterol-lowering soluble fiber, legumes such as tender, white cannellini beans are slow to raise blood sugar. As part of a 2012 University of Toronto study, 121 people with type 2 diabetes followed a healthy diet containing a daily cup of beans or whole grains. After three months, the bean group saw their A1c levels - a check of average blood sugar levels - fall nearly twice as much as the whole-grain group.

\section{Spinach}

Spinach is one of many leafy greens that have been shown to drop the risk of developing diabetes; collards are another great choice. People who consume more than one serving a day of spinach and other leafy greens slashed their risk by 14 percent, compared to people who ate less than $1 / 2$ a serving daily, found one British study. This green is particularly rich in vitamin $\mathrm{K}$, along with several minerals including magnesium, folate, phosphorus, potassium, and zinc. It's also a good source of the plant chemicals lutein and zeaxanthin, and various flavonoids. Although spinach is technically a rich source of calcium, another nutrient in spinach called oxalic acid prevents much of that calcium from being absorbed, but you can blanch spinach (boil it for just one minute) to reduce this chemical.

\section{Sweet potatoes}

One analysis found that sweet potatoes reduce $\mathrm{HbAlc}$ measures between 0.30 and 0.57 percent and fasting blood glucose by 10 to 15 points. Sweet potato also contains anthocyanins, which are the natural pigments that give the sweet potato its deep orange color and the antioxidants believed to have anti-inflammatory, antiviral, and antimicrobial qualities.

\section{Walnuts}

The most widespread tree nut in the world, walnuts contain the polyunsaturated fatty acid called alpha-linolenic acid, which has been shown to lower inflammation. The L-arginine, omega-3s, fiber, vitamin E, and other phytochemicals found in walnuts and other tree nuts make them potent: scientists have found them to have antioxidant, anticancer, antiviral, and anti-high cholesterol actions. These powers can help stop and reverse the progression of chronic conditions such as diabetes and heart disease. Snack on walnuts in their shells; the time it takes to crack them open can help you slow down, so your body has more time to register the food and you feel full with fewer calories.

\section{Quinoa}

Quinoa tastes like a grain, but it's more closely related to spinach than it is to rice. Contrary to most grains, quinoa is a dense source of "complete" protein (14 grams per $1 / 2$ cup!), boasting all nine essential amino acids. One is lysine, which helps the body absorb all that fat-burning calcium and also helps produce carnitine, a nutrient responsible for converting fatty acids into energy and helping to lower cholesterol. One of the most fiber-rich grain-like foods, quinoa contains 2.6 grams per $1 / 2$ cup, and fiber helps to balance blood sugar levels and keep you fuller, longer.

\section{Cinnamon}

Several studies show that this delicious spice can help reduce blood sugar. One, published in the journal Diabetes Care, noted how people with type 2 diabetes who'd taken one or more grams of cinnamon daily had dropped their fasting blood sugar by a whopping 30 percent, compared to people who took no cinnamon. They also reduced their triglycerides, LDL cholesterol, and total cholesterol by upwards of 25 percent. Here's why: Cinnamon is rich in chromium, a mineral that enhances the effects of insulin. It's also loaded with polyphenols, antioxidants that gather up all the free radicals in your blood to protect you from cancer and also lower systemic inflammation, further guarding you from diabetes and heart disease.

\section{Collard greens}

Dark green leafy vegetables like collard greens are excellent sources of vitamin $\mathrm{C}$, which helps lower cortisol in the body and consequently reduces inflammation as well. Collard greens (and other cruciferous veggies like kale and Brussels sprouts) are also a good source of alpha-lipoic acid (ALA), a micronutrient that helps the body deal with stress. When scientists at the Linus Pauling Institute at Oregon State University gave aging rats ALA, they found that the animals' bodies created their own antioxidants, making them better able to resist toxins in the environment, and to reduce inflammation. Good news for diabetes: ALA also helps reduces blood sugar and can help to strengthen the nerves damaged by diabetic neuropathy. Just be careful not to overcook it, which creates a strong sulfur smell. Just five minutes of steaming, and you're done.

\section{Turmeric}

Turmeric may have been protecting the health of an entire Indian subcontinent for about 5,000 years. A traditional Indian diet features white rice and flour breads, which as rapidly digested carbs would ordinarily raise blood sugar dramatically. But the presence of turmeric - the yellow spice that lends its color to many curry dishes - helps to manage the potent impact on blood sugar. Curcumin, the active ingredient in turmeric, is the compound believed to regulate fat metabolism in the body. Curcumin acts directly on fat cells, pancreatic cells, kidney cells, and muscle cells, dampening inflammation and blocking the nefarious activities of cancer-causing tumor necrosis factor and interleukin-6. Experts believe the combined action of all of these factors together gives curcumin the power to reverse insulin resistance, high blood sugar and high cholesterol, and other symptoms linked to obesity. 